\section{Convergence of drug action}

\section{Robert B. Freedman}

Two papers on pages 755 and 758 of this issue $^{1.2}$ add a further intriguing twist to the emerging connection between immunosuppressant drugs, the cytosolic proteins that bind them and the enzymatic activity of those proteins. Earlier this year, Fischer et al. ${ }^{3}$ and Takahashi et al. ${ }^{4}$ showed that the protein, cyclophilin, which binds the immunosuppressant drug cyclosporin A with high affinity and is assumed to mediate its action, has prolyl-peptidyl cistrans isomerase (PPIase) activity. Their work also showed that cyclophilin is identical to a previously identified PPIase ${ }^{5}$, and that the PPIase activity is specifically inhibited by cyclosporin. Now, two groups that have been exploring the action of the structurally distinct immunosuppressant, FK506, report ${ }^{1,2}$ that it, too, is bound by a specific cytosolic binding protein with PPIase activity, but that this binding protein is quite distinct from cyclophilin.

The two groups, Siekierka et al. ${ }^{1}$, at Merck, Sharp and Dohme, and Harding et $a l .^{2}$, at Harvard and Yale Universities, were following up the finding (see ref. 6) that the macrolide FK506 is a distinctly more potent immunosuppressant than the cyclic peptide, cyclosporin, which is widely used for prevention of graft rejection in organ transplantation. Siekierka $e t$ al., having previously shown that FK506 bound to a cytosolic protein ${ }^{7}$, purified this protein from the JURKAT T-cell line. Harding et al. have synthesized affinity matrices containing cyclosporin-and FK506-derived ligands for affinity chromatographic purification of the corresponding binding proteins from human and bovine tissues. Both groups found that the purified FK506-binding protein is distinct from cyclophilin in terms of size and $\mathrm{N}$-terminal sequence (and, indeed, is unrelated to any sequence in the current database). Both groups used radioligand binding assays and competitive binding studies to characterize the ligand binding activity; Siekierka and colleagues found that FK506 binds to its protein with a 1:1 stoichiometry, and an affinity close to 1 nanomolar.

Most importantly, both groups demonstrate that the purified FK506-binding protein has PPIase activity, although the activity towards the conventional PPIase substrate (a substituted proline-containing tetrapeptide) is 20-25-fold lower than that of cyclophilin. And finally the groups show that cyclophilin and the FK506-binding protein are quite distinct in terms of specificity; cyclophilin binds, and is inhibited by cyclosporin and shows no recognition of FK506, whereas the converse holds for the FK506-binding protein. This difference in specificity is seen most clearly

\section{ASTRONOMY}

\section{R. F. Carswell}

in the kinetic results of Siekierka et al., which show complete inhibition of each receptor by its corresponding ligand at concentrations where the other drug has no effect whatsoever.

Minor differences between the results reported in the two papers indicate that the story is far from complete. The binding protein purified by Siekierka et al. has a relative molecular mass $\left(M_{\mathrm{r}}\right)$ of 11,000 and shows saturable ligand binding at concentrations in the nanomolar range, whereas the protein purified by Harding $e t$ al. has a $M_{\mathrm{r}}$ of 14,000 and is not saturated at ligand concentrations up to 1 micromolar. Although these discrepancies may derive from the fact that the proteins come from different species, and from the use of distinct FK506 derivatives as radiolabelled ligands, possibly a diverse family of proteins is involved. Siekierka and colleagues do not report any sequence for their protein, so it is not certain that the proteins purified by the two groups are identical.

In the light of earlier data, which indicate that FK506 and cyclosporin act on T-lymphocytes in essentially equivalent fashion $^{8}$, the new data suggest that the two drugs act through distinct pathways but that their modes of action converge on PPIases. Current data show that these proteins are abundant and are important in mediating the activation of $\mathrm{T}$ cells, but how and why this activation exploits their ability, as PPIases, to catalyse rotamerization of peptide bonds to prolyl residues, remains completely mysterious. Far less mysterious is the fact that this new clue to the action of a class of drugs with multimillion-dollar sales, is now generating vigorous research activity.

Professor Robert B. Freedman is in the Biological Laboratory, University of Kent, Canterbury, Kent GT2 7NJ, UK.

1. Siekierka, J.J., Hung, S.H.Y., Poe, M., Lin, C.S. \& Sigal, N.H. Nature 341, 755-757 (1989).

2. Harding, M.W., Galat, A., Uehling, D.E. \& Schreiber, S.L Nature 341, 758-760 (1989)

3. Fischer, G., Wittmann-Liebold, B., Lang, K., Kiefhaber, T. \& Schmid, F.X. Nature 337, 476-478 (1989).

4. Takahashi, N., Hayano, T. \& Suzuki, M. Nature 337, 473475 (1989).

5. Lang, K., Schmid, F. X. \& Fischer, G. Nature 329, 268270 (1987).

6. Thomson, A.W. Immunology Today 10, 6-9 (1989)

7. Siekierka, J.J., Staruch, M., Hung, S.H.Y. \& Sigal, N.H. J. Immun. (in the press)

8. Tocci, M. J. et al. J. Immun.(in the press)

\title{
Galactic chemical evolution
}

Distant galaxies, that is those at high redshift, are seen as they were at a much earlier epoch in the history of the Universe. From their forms and distributions, it is hoped to learn what unseen effects in earlier epochs led to their formation. From their spectra, it is hoped to learn their chemical compositions which, in turn, should tell us about the progress of stellar nucleosynthesis in the early Universe. Two groups now report ${ }^{1,2}$ the measurements of the spectral lines of zinc in galaxies illuminated by bright background quasars. Because zinc is one of the few elements that do not become bound into interstellar grains (where they would be invisible), these spectra give us the best measure yet of early nucleosynthesis.

Element synthesis in the primordial fireball during the first few thousand seconds of the Universe yielded almost exclusively isotopes of hydrogen and helium. All the other elements, referred to as the heavy elements, were made later in stars after they and galaxies coalesced out of the initial material distribution. The heavy-element enrichment of the interstellar medium in our Galaxy and in others occurs when the processed materials from stars is ejected, either relatively slowly through stellar winds or explosively if the star becomes a supernova. This medium partakes in subsequent star formation, nuclear burning and further enrichment of the interstellar medium.

Information on the details of the chemical history of our Galaxy has been obtained from studies of the heavyelement abundancies in the atmospheres of stars for which age estimates are available. The overall picture is that some initial enrichment of elements such as carbon, nitrogen and oxygen occurs relatively quickly from processing in shortlived, high-mass stars. After $10^{9}$ years or so the iron-group elements appear in the interstellar medium when longer-lived, lower-mass stars yield significant quantities of material. The rate of enrichment depends on the relative numbers of stars at various masses and their formation rate, the fraction of the stellar ejecta which escapes from the Galaxy completely, and any accretion of primordial material into the Galaxy.

An independent study of the chemical history of galaxies can be made by analysing the element abundances at high redshifts when they (and the Universe) were much younger. At present, the only way of doing this with any reliability is to examine the spectra of bright highredshift quasars for absorption lines from hydrogen and heavy elements which arise 